ECCOMAS

Proceedia
COMPDYN 2021

$8^{\text {th }}$ ECCOMAS Thematic Conference on Computational Methods in Structural Dynamics and Earthquake Engineering M. Papadrakakis, M. Fragiadakis (eds.) Streamed from Athens, Greece, 28 - 30 June 2021

\title{
NONLINEAR FINITE ELEMENT MODELS FOR EMBEDDED BASE CONNECTIONS
}

\author{
Nicolas Mora-Bowen ${ }^{1}$, Pablo Torres-Rodas ${ }^{2}$ \\ ${ }^{1}$ Universidad San Francisco de Quito \\ Diego de Robles \\ e-mail: nmorabowen@estud.usfq.edu.ec \\ ${ }^{2}$ Universidad San Francisco de Quito \\ Diego de Robles \\ e-mail: patorresr@usfq.edu.ec
}

\begin{abstract}
Column base connections are one of the most critical components in Steel Moment Frames (SMFs) since these connections transfer the loads (i.e., gravity, seismic, wind) from the entire superstructure into the concrete-foundation, being an interface between them. Typically, exposed base plates are preferred for low-and mid-rise buildings, while embedded base connections are the norm for tall buildings. This latter base configuration response is controlled by complex interactions between the column flange and the bottom base plate with the concrete foundation, where the mechanisms to transfer internal forces are idealized to underpin the current strength design methods. These mechanisms include horizontal bearing stresses between the column flange and the surrounding concrete and vertical bearing stresses of the base plate and the concrete foundation. Current methods to estimate the strength of these connections are validated against a limited number of experimental tests complicating their generalization for the different configurations that have not been tested. Although the results from these methods show good agreement with test data, the assumptions that underpin these methods have not been verified through sophisticated nonlinear finite element models. Motivated by this issue, this paper presents a series of nonlinear finite element models developed to provide insights into the behavior of embedded base connections for SMFs. These models incorporate the essential aspects that control the connection behavior, including constitutive material modeling and contacts among the connection components. Possible design implications are discussed, while the limitations of the current work and future lines of research are outlined.
\end{abstract}

Keywords: Column Base Connections, Embedded Base Connections, Finite Element Models. 


\section{INTRODUCTION}

Column Base Connections are one of the most important components of Steel Moment Frames (SMFs). Several researchers [1-6] have demonstrated their significant influence on the seismic performance of SMFs systems. Because of this, these connections have been extensively studied over the last decade in different programs [7-11]. Typically, base connections can be broadly classified as exposed base plates (EBPs) and embedded base connections (EmBCs). The former type is the preferred detail for low to mid-rise buildings, while the latter is the norm for tall buildings.

EmBCs consist of a column welded to a bottom base plate (Refer to Fig. 1) and embedded into a concrete foundation. The applied forces, i.e., Axial Load, Bending Moment, and Shear, are resisted by a combination of the bearing stresses developed by the contact between the column flange and the foundation and by the vertical stresses at the bottom base plate. Grilli and Kanvinde [7] conducted a large-scale experimental program to study the seismic response of EmBCs. This program's focus was the flexural capacity of these connections and the development of a strength method based on the insights gained from the tests. A total of five tests were evaluated. The difference among them was the embedded length (510 and 760mm), column size, and axial load level.

Grilli and Kanvinde [7] postulated an internal stress distribution to idealize the mechanism that resists the applied loads. Figure 1 illustrates this mechanism which consists of horizontal bearing stresses accompanied by joint shear in the panel zone and vertical stresses at the bottom base plate. However, this idealized mechanism relies on pre-defined stress distribution (rectangular) on the bottom base plate as well as in the column flange against the foundation. It is wellknown that the real stress distribution might differ from the assumed one due to the complex interactions between the components.

The behavior of EmBCs has been studied in the past from different perspectives. Grilli and Kanvinde [7] suggested a strength method to estimate their flexural strength. Torres-Rodas [12] proposed a procedure to estimate their rotational stiffness by aggregating the deformations within the components. The hysteretic characteristics of these connections were explored by Torres-Rodas [13] through a hinged model, which was validated experimentally and capture the force transfer mechanisms observed at [7]. Recently, Inamasu et al., [14] developed Finite Element (FE) models to explore the behavior of EmBCs further and suggest design recommendations. These models consist of shell elements with two rotational springs. Results indicate that current methods for the design of EmBCs are nonconservative.

Motivated by the preceding discussion, this paper presents a study with 3D sophisticated FE models to investigate further the seismic behavior of EmBCs. These models provide insights into the internal stress distribution and patterns of deformation of these connections. The models intend to capture phenomena such as the multi-axial constitutive response of the materials (steel and concrete), large deformations, and the contact between the column flange and the foundation. The paper starts by describing the models developed herein, and then the main findings are presented. Finally, the limitations of the study are discussed, and recommendations for future research are presented. 


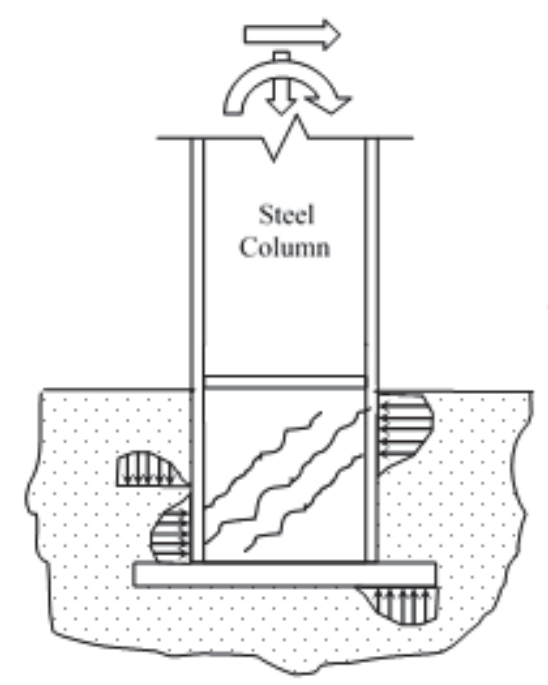

Figure 1: EmBC detail and internal force mechanism (from [7,12])

\section{EXPERIMENTAL DATA}

In this study, for the purposes of model validation, the experimental program conducted by Grilli and Kanvinde [7] at the University of California Davis was used. Thus, in this section of the paper, the details of the experimental program are briefly summarized. All tests consist of a steel column welded to a bottom base plate. The column height was about $3 \mathrm{~m}$, and it was embedded into a concrete foundation. All the specimens were subjected to the cyclic loading protocol ATC-SAC (Krawinkler et al. 2000 [15]). The parameters that changed among the tests are the embedded depth, the level of Axial Load (Tensile forces were used in one test), and the column size. Table 1 shows a summary of the test parameters, while Figure 2 a schematic representation of the test setup at UC Davis.

Based on the insights obtained from the mentioned experimental program, Grilli and Kanvinde [7] presented a strength method to characterize the flexural capacity of EmBCs. Key aspects of this method are detailed here. The applied Moment is resisted by a combination of horizontal bearing stresses developed by the contact between the column flange and the concrete foundation and vertical bearing stresses at the bottom base plate. Shear forces in the panel zone complement the horizontal bearing stresses. Thus, the Moment is resisted by these two mechanisms, i.e., horizontal and vertical stresses, with the implication that a fraction of the total applied Moment is distributed to each mechanism. This idealization entails an indeterminate problem, which is solved by the introduction of an empirically calibrated equation that considers the relative stiffness of the embedded column and the surrounding media.

In this manner, the connection strength may be calculated based on the associated limit states to each force transfer mechanism. For the horizontal bearing stresses, two limit states can take place 1) concrete bearing failure and 2) joint shear failure. On the other hand, in the vertical bearing mechanisms, four possible modes of failure are identified 1) base plate yielding, 2) concrete breakout under the base plate, 3 ) concrete breakout above the base plate, and 4) bearing failure of the concrete around the base plate. The connection strength is calculated by combining the Moment capacity associated with both mechanisms $\left(M_{H B}^{\text {capacity }}\right.$ and $\left.M_{V B}^{\text {capacity }}\right)$. Grilli and Kanvinde [7] concluded that the capacity estimated with the preceding procedure must be reduced by $30 \%$ for design purposes. 


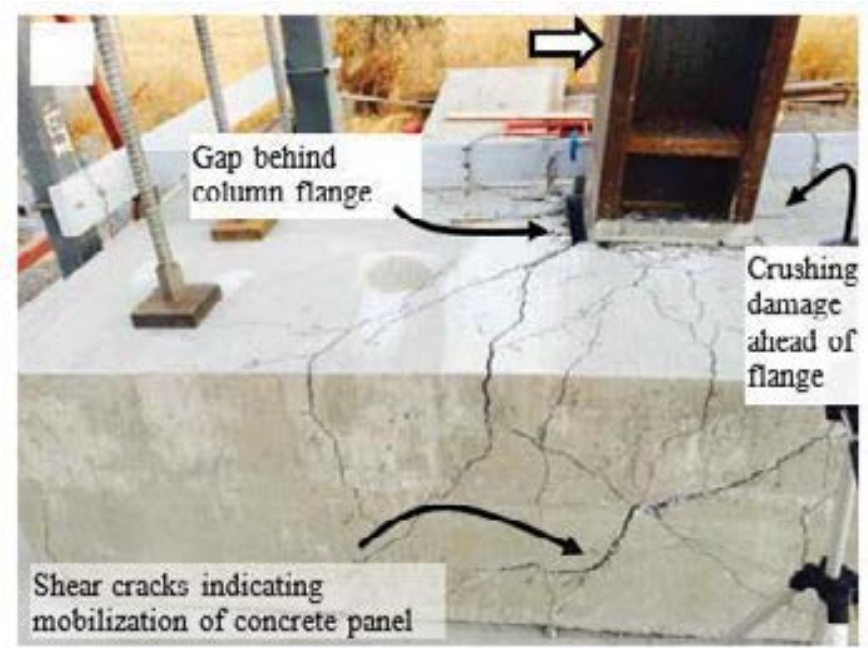

Figure 2: UC Davis experimental test (from [7,12])

\section{FINITE ELEMENT MODELS}

A total of four FE models have been developed to study the seismic behavior of EmBCs using the ABAQUS [16] simulation platform. Figure 3 illustrates a representative model built in this investigation. The models are composed of 3D Hex-structured elements. The meshes were refined in places where concentrations of stress are anticipated. As described in the previous section, the strength of the connection may be explained due to the contacts between the column flange and the base plate with the concrete foundation. Thus, the contacts are an essential feature of these models. These contacts are simulated with a finite sliding formulation with Normal and Tangential interaction properties. The former property was defined as hard contact, while the latter with a frictional formulation following the penalty method. A friction coefficient of 0.45 is adopted as recommended by Gomez et al. [11]. In contrast, welded elements inside the connection are assigned the tie constraint (i.e., steel column and base plate, steel column, and stiffeners) since welds are detailed to resist fracture even at large deformations.

Geometric nonlinearities, including large deformations formulations, are included in the models. The steel column and base plate are modeled with the Von-Misses surface with isotropic hardening. On the other hand, for concrete modeling, it is common to assume that this material behaves as an elastoplastic material in compression and brittle in tension. In this investigation, the concrete damaged constitutive plasticity (CDP) model was employed since it provides a general capability for modeling concrete and other quasi-brittle materials. Some of the details of this constitutive model provided by [16] are discussed. The CDP model uses concepts of isotropic damaged elasticity in combination with isotropic tensile and compressive plasticity to represent the inelastic behavior of concrete. It assumes that the main two failure mechanisms are tensile cracking and compressive crushing of the concrete material. Two hardening parameters control the evolution of the yield surface, $\epsilon_{t}^{-p l}$ and $\epsilon_{c}^{-p l}$ associated to failure mechanisms under tension and compression loading, respectively [16]. The material properties are obtained from the ancillary tests from [7], and true stresses and strains were assumed in the material formulation.

The FE models were subjected to the SAC load protocol [15] in the Axial Load presence. In order to avoid the interference of P-delta effects in the connection response, the Axial Load was 
applied strategically at the bottom of the base plate. Thus, the Moment-Rotation response was recorded from the simulations and compared to validate the models with the associate experimental test. Figure 4 illustrates the results of the simulations and the corresponding validation against the experimental data. As per this Figure, the essential aspects of the hysteretic response are capture by the numerical results. Specifically, the simulations are able to capture the pinching behavior observed in the tests. Cyclic deterioration of the strength and the unloading stiffness are identified in these connections and well-represented in the simulations. The parameters that define the response's backbone curve are the Moment at First Yield, the Initial Rotational Stiffness, the Peak Moment Strength, and the Rotation Associate to the Peak Strength. A visual inspection of Figure 4 indicates that the differences between the simulations' parameters to the experimental tests' corresponding values are neglectable. Consequently, in the authors' opinion, the FE models are able to capture the key features of the connection response and are appropriate to investigate the behavior of EmBCs.
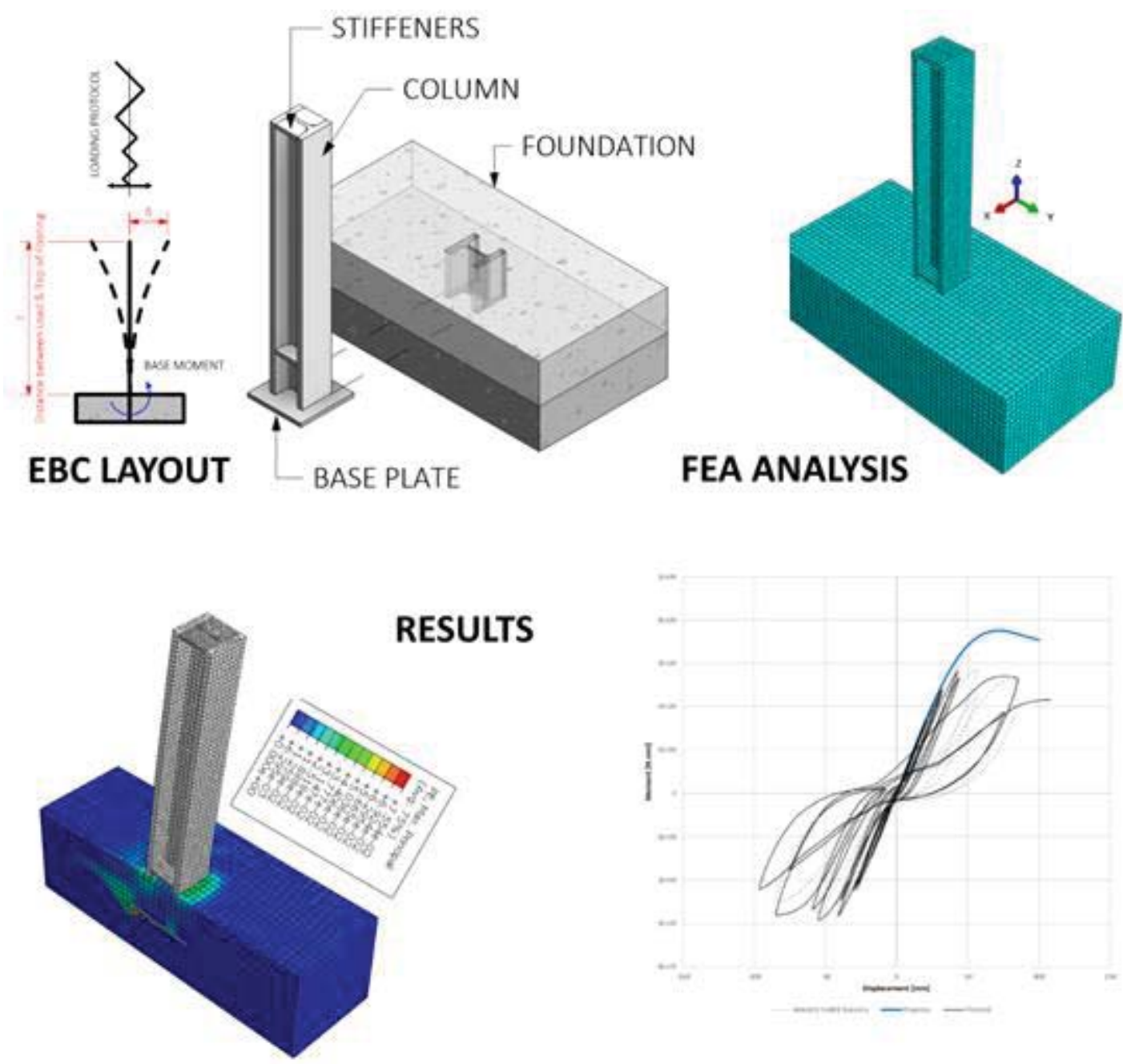

Figure 3: a) EmBC layaout, b) representative 3D FE model, c) example of the distribution of Stresses of a simulation, d) example of a hysteretic response 


\begin{tabular}{cccccccc}
\hline & & \multirow{2}{*}{$d_{\text {embeded }}$} & \multicolumn{5}{c}{ Base Plate } \\
\cline { 5 - 8 } Test \# & Column Size & $P$ & $t_{p}$ & $N$ & $B$ & $Z$ \\
\cline { 3 - 7 } & & {$[\mathrm{kN}]$} & {$[\mathrm{mm}]$} & {$[\mathrm{mm}]$} & {$[\mathrm{mm}]$} & {$[\mathrm{mm}]$} & {$[\mathrm{mm}]$} \\
\hline 1 & $\mathrm{~W} 14 \times 370$ & $445[\mathrm{C}]$ & 510 & 50 & 760 & 760 & 2850 \\
2 & $\mathrm{~W} 18 \times 311$ & $445[\mathrm{C}]$ & 510 & 50 & 865 & 710 & 2850 \\
\hline 3 & $\mathrm{~W} 14 \times 370$ & 0 & 760 & 50 & 760 & 760 & 3100 \\
4 & $\mathrm{~W} 14 \times 370$ & $445[\mathrm{C}]$ & 760 & 50 & 760 & 760 & 3100 \\
\hline
\end{tabular}

Table 1: Test Matrix Geometry and applied Axial Loads

\section{DISCUSSION OF THE RESULTS}

This section discusses the key findings from the FE simulations. Referring to Figure 4, all simulations show a well-defined linear-elastic branch up to the point where nonlinear behavior starts, which corresponds to rotations close to $0.005 \mathrm{rad}$. This observation is consistent with the experimental evidence reported by [7] that indicates that after $0.005 \mathrm{rads}$, small cracks on the tension column flange start to open, entailing a gradual nonlinear response. These small cracks are accompanied by diagonal cracks, which start to grow near the steel column corners. Figure 5 shows a schematic representation of the crack propagation obtained from the simulation of test 1 once the deformation progresses.

As deformation progresses in all simulations, an appreciable loss of stiffness is observed because the concrete reaches its peak strength. At this point, the gap formed in the foundation in the tension flange side grows, which is consistent with the experimental tests [7]. This gap entails the pinching behavior observed in all the tests since it causes a relatively unconstrained rotation of the steel column as it moves back to the vertical position and goes in the reverse direction (following the cyclic loading protocol). A closer inspection of Figure 4 reveals that tests with higher embedment depths $(760 \mathrm{~mm})$ present a more gradual stiffness decrease. This phenomenon may be attributed to the fact that higher embedment lengths imply a great fraction of the total Moment carried by the horizontal bearing stresses. Thus, when this latter mechanism starts to deteriorate, the force transfer to the vertical stresses seems to be more gradual.

Finally, in this section, the distribution of the horizontal bearing stresses is discussed. Figure 6 shows the stress distribution along the steel column flange of test 1 in two stages of the response, i.e., at the Moment associated with the First Yield and Peak Strength. As per Figure 6, for the first condition (i.e., My), the "real" stress distribution is closer to a triangular shape. This observation is not surprising since at this level of deformation, the connection starts to incursion in the nonlinear regime, and a triangular shape is consistent with an elastic behavior. In contrast, once the connection has reached its peak, it is observed in the simulations a more uniform stress distribution along a certain length of the flange. This finding is consistent with the rectangular stress block assumed by Grilli and Kanvinde [7] in their strength method. Although these findings may be used in the future to refine the strength, this topic deserves further scrutiny. 

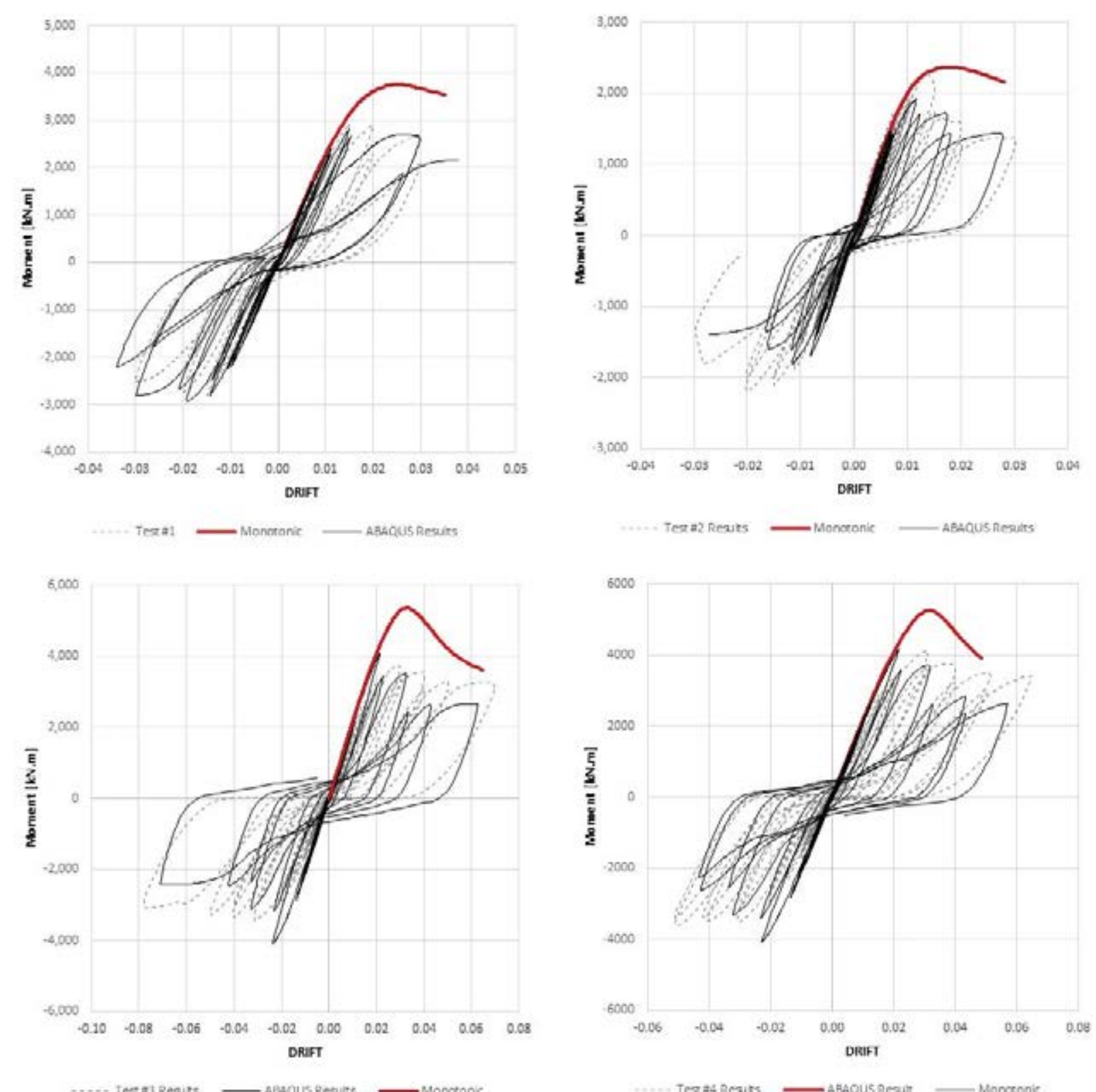

Figure 4: Hysteretic response of all FE simulations vs. Experimental data from [7]

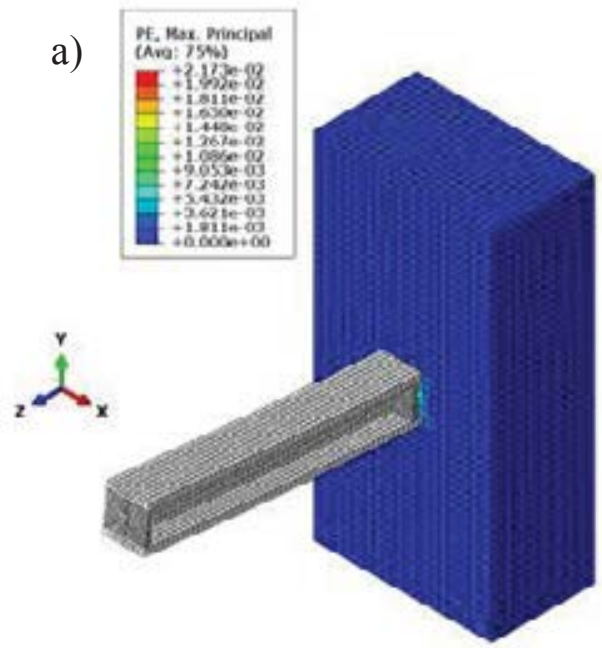

b)

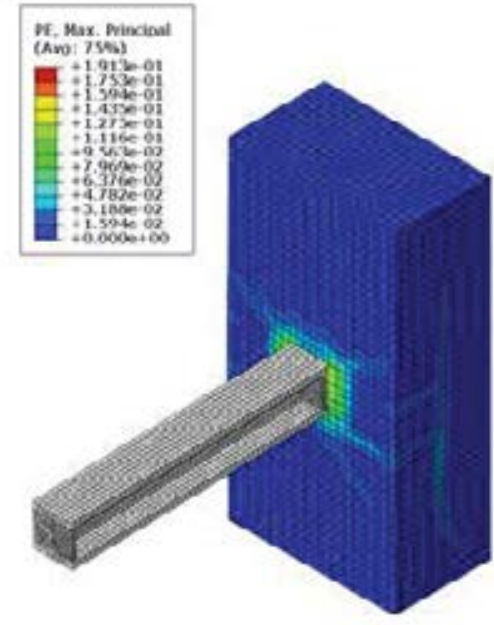

Figure 5: plastic strain distribution on the concrete at a) first yield, b) peak strength 
a)

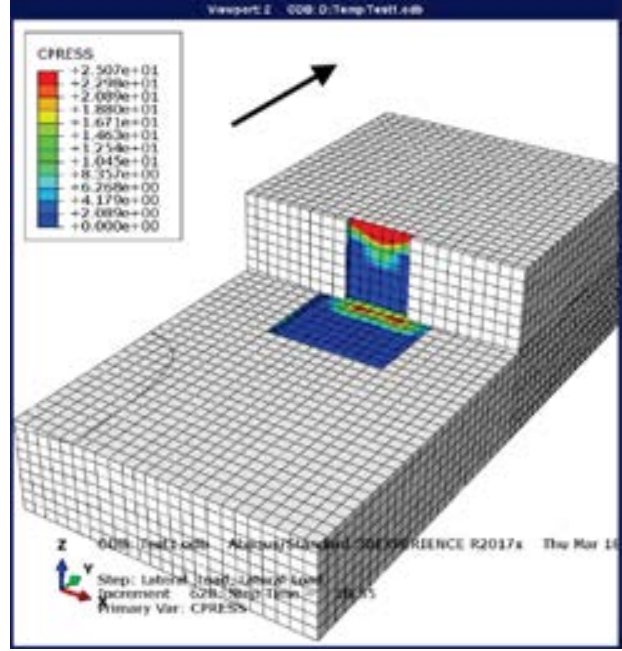

b)

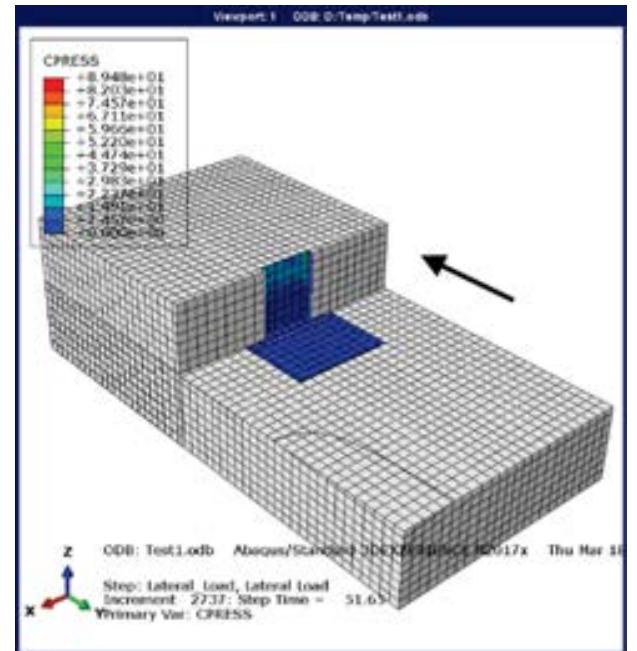

Figure 6: stress distribution on the concrete foundation due to column flange contact at rotations associated with a) first yield, b) peak strength

\section{SUMMARY, LIMITATIONS, AND RECOMMENDATIONS}

This paper presents a numerical study conducted on a critical component of SMFs such as EmBCs. A total of four FE models were developed in the ABAQUS platform [16] to explore the seismic behavior of these connections. Specifically, in this study, the internal force distribution and the patterns of deformation were identified from the simulations with the intention to gain insights aimed to improve the current design methods (e.g., [7]). All the models were validated against the experimental program conducted by Grilli and Kanvinde [7]. The goodness of the fit (i.e., the difference between the experimental and the numerical results) was considered an indicator of the reliability of the models.

The simulations indicate that at rotation levels close to the "first" yield of the connection, the stresses are not uniform along the column flange; instead, a triangular shape can be assumed. In contrast, at higher rotations, the stresses tend to become relatively uniform. Thus, when the connection reaches its peak strength, it seems that the rectangular block assumption is appropriate. However, this issue deserves a further look.

The paper has several limitations that should be addressed in a new study in order to generalize the conclusions. For example, the number of simulations (four in total) is limited. Moreover, the experimental program by [7] is the only testbed used. It is recommended to build more FE models from other experimental programs available to assess different configurations' behavior (e.g., column size, embedment length, the influence of shear studs). Besides, this study does not address issues related to the seismic demands on the EmBCs, or a reliability analysis to assess if the strength resistance factors adopted are adequate or not. These topics have been addressed for exposed-based plates by $[17,18,19]$ and should be conducted for EmBCs. 


\section{REFERENCES}

[1] Torres-Rodas, P., Flores, F., \& Zareian, F. (2018). Seismic response of steel moment frame considering gravity system and column base flexibility. In Proc. 11th US Natl. Conf. Earthq. Eng., June 25-29, Los Angeles, USA.

[2] Falborski, T., Torres-Rodas, P., Zareian, F., \& Kanvinde, A. (2020). Effect of baseconnection strength and ductility on the seismic performance of steel moment-resisting frames. Journal of Structural Engineering, 146(5), 04020054.

[3] Torres-Rodas, P., Flores, F., Astudillo, B.X., Pozo, S. (2020). Sensitivity of special steel moment frames to the influence of column-base hysteretic behavior including gravity framing system. Proceedings of the International Conference on Structural Dynamic , EURODYN, 2020, 2, pp. 3629-3642

[4] Inamasu, H., de Castro e Sousa, A., Güell, G., \& Lignos, D. G. (2020). Anchor-yield exposed column bases for minimizing residual deformations in seismic-resistant steel moment frames. Earthquake Engineering \& Structural Dynamics.

[5] Y. Cui, F. Wang, S. Yamada, Effect of Column Base Behavior on Seismic Performance of Multi-Story Steel Moment Resisting Frames, International Journal of Structural Stability and Dynamics. 19 (2019) 1940007. https://doi.org/10.1142/S0219455419400078.

[6] Torres-Rodas, P., Flores, F., Pozo, S., \& Astudillo, B. X. (2021). Seismic performance of steel moment frames considering the effects of column-base hysteretic behavior and gravity framing system. Soil Dynamics and Earthquake Engineering, 144, 106654.

[7] Grilli, D.A. and Kanvinde, A.M. (2015). "Embedded Column Based Connections subjected to Flexure and Axial loads," Report 3-11 submitted to the Charles Pankow Foundation.

[8] DeWolf J.T., and Sarisley, E.F. (1980), "Column Base Plates with Axial Loads and Moments," Journal of the Structural Division, ASCE, Vol. 106, No. 11, November 1980, pp. 2167-2184

[9] Astaneh, A., Bergsma, G., and Shen J.H. (1992). "Behavior and Design of Base Plates for Gravity, Wind and Seismic Loads," Proceedings of the National Steel Construction Conference, Las Vegas, Nevada, AISC, Chicago, Illinois.

[10] Burda, J.J., and Itani, A.M. (1999). "Studies of Seismic Behavior of Steel Base Plates," Report No. CCEER 99-7, Reno (NV): Center of Civil Engineers Earthquake Research, Department of Civil and Environmental Engineering, University of Nevada, NV.

[11] Gomez I.R., Kanvinde A.M. and Deierlein G.G. (2010). "Exposed Column Base Connections Subjected to Axial Compression and Flexure," Report Submitted to the American Institute of Steel Construction (AISC), Chicago, IL.

[12] Rodas, P. T., Zareian, F., \& Kanvinde, A. (2017). Rotational stiffness of deeply embedded column-base connections. Journal of structural engineering, 143(8), 04017064.

[13] Torres-Rodas, P., Zareian, F., \& Kanvinde, A. (2018). A hysteretic model for the rotational response of embedded column base connections. Soil Dynamics and Earthquake Engineering, 115, 55-65. 
[14] Inamasu, H., Kanvinde, A. M., \& Lignos, D. G. (2021). Seismic design of non-dissipative embedded column base connections. Journal of Constructional Steel Research, 177, 106417.

[15] Gupta, A., \& Krawinkler, H. (2002). Prediction of Seismic Demands for SMRF's with Ductile Connections and Elements. SAC Joint Venture.

[16] ABAQUS, ABAQUS Analysis User's Manual Version 6.14-1, Dassault Systems Simulia Corp, RI, USA, 2014.

[17]Torres-Rodas, P., Zareian, F., \& Kanvinde, A. (2018). Seismic demands in column base connections of steel moment frames. Earthquake Spectra, 34(3), 1383-1403.

[18] Torres-Rodas, P., Fayaz, J., \& Zareian, F. (2020). Strength resistance factors for seismic design of exposed based plate connections in special steel moment resisting frames. Earthquake Spectra, 36(2), 537-553.

[19] Song, B., Galasso, C., \& Kanvinde, A. (2021). Reliability Analysis and Design Considerations for Exposed Column Base Plate Connections Subjected to Flexure and Axial Compression. Journal of Structural Engineering, 147(2), 04020328. 